\title{
Politique
}

\section{Les transformations du corps médical québécois, 1960-1980 : adaptation d'un monopole professionnel à la domination légale-rationnelle?}

\section{Denise Couture}

Numéro 12, automne 1987

Mouvements et acteurs

URI : https://id.erudit.org/iderudit/040567ar

DOI : https://doi.org/10.7202/040567ar

Aller au sommaire du numéro

Éditeur(s)

Société québécoise de science politique

ISSN

0711-608X (imprimé)

1918-6584 (numérique)

Découvrir la revue

Citer cet article

Couture, D. (1987). Les transformations du corps médical québécois, 1960-1980 : adaptation d'un monopole professionnel à la domination légale-rationnelle? Politique, (12), 37-62. https://doi.org/10.7202/040567ar d'utilisation que vous pouvez consulter en ligne. 


\title{
LES TRANSFORMATIONS DU CORPS MÉDICAL QUÉBÉCOIS, 1960-1980: ADAPTATION D'UN MONOPOLE PROFESSIONNEL À LA DOMINATION LÉGALE-RATIONNELLE?
}

\author{
Denise Couture* \\ Université de Montréal
}

\section{Introduction}

Cet article porte sur les transformations qui se sont produites au sein du corps médical québécois depuis le début des années 60 et sur la possibilité d'expliquer ces transformations en termes de l'adaptation d'un monopole professionnel à la domination légalerationnelle. Il repose sur l'analyse d'un ensemble de données qui ne pourront malheureusement pas être exposées systématiquement ici. Mentionnons seulement que ces données consistent surtout en documents tels que, parmi les principaux, la revue publiée par la Corporation professionnelle des médecins du Québec, des textes législatifs et réglementaires, le recueil des décisions disciplinaires prises par les comités de discipline des corporations professionnelles et publié par l'Office des professions du Québec,

* Je tiens à remercier Brigitte Dumas et les évaluateurs anonymes pour leur critique de ce texte.

Politique, 12 (Automne 1987). 
diverses études sur les effectifs médicaux et les modes de pratique de la médecine au Québec de même que les procès-verbaux des réunions des comités d'évaluation médicale et dentaire d'un centre hospitalier universitaire sur une période d'un an.

La thèse principale qui sera avancée à partir de l'examen de ces matériaux, concerne le sens, la signification, la portée des changements qui sont survenus au sein du corps médical québécois depuis le début des années 60. Plus précisément, étant donné qu'une bonne partie de ces changements ont été provoqués par les réformes et interventions de l'État québécois dans le domaine de la santé, cette thèse concerne les rapports entre l'évolution de l'État québécois et l'évolution de la profession médicale au Québec. Elle est en résumé la suivante: en dépit du discours anti-étatique parfois véhément tenu par la profession médicale au Québec, cette profession doit une bonne partie du maintien de son statut au sein de la société québécoise, aux interventions et réformes de divers gouvernements depuis deux décennies. En effet, la construction de l'identité de l'État québécois comme État moderne à travers une série d'interventions qui ont contribué à le constituer comme interlocuteur principal dans bon nombre de rapports sociaux, cette construction d'identité, donc, a été une condition essentielle du renouvellement des bases de légitimation de la place et de l'action de la profession médicale au sein de la société québécoise. À la limite, on pourrait aller jusqu'à affirmer la chose suivante: si le corps médical québécois demeure puissant, c'est en bonne partie grâce aux interventions parfois autoritaires de l'État québécois qui ont permis à cette profession de développer sa capacité d'argumenter en fonction d'une «rhétorique de la place publique» et de se mettre au diapason du reste de la société québécoise en instance de modernisation.

De plus, nous prétendons que cette adaptation parfois forcée du corps médical québécois s'est faite principalement à travers la 
création et la protection des droits individuels - les individus concernés ici étant surtout les médecins pris un à un - protection qu'ont assurée la réforme de la santé et la réforme du droit professionnel. Voilà un angle sous lequel ces réformes ont été peu examinées. Cette ligne d'interprétation se rapproche de celle développée par Pierre Rosanvallon (1981) qui, cherchant à expliquer la Crise de l'État-Providence, remonte à ses origines sous la forme d'un État-protecteur ${ }^{1}$.

"Au départ, une reconnaissance intellectuelle décisive: celle du lien qui unit historiquement le développement de l'État à celui de l'individu. Pas d'Étatprotecteur pensable et possible sans l'émergence de l'individu comme catégorie politique et juridique. (...)

L'État-providence ne fait que poursuivre et étendre ce mouvement de protection de l'individu comme figure centrale du social. Son objectif est de le dégager des réseaux de solidarité contraignants, aléatoires dans lesquels il s'insérait encore: L'État-providence veut libérer l'individu en simplifiant le social [en italiques dans le texte]. Il s'agit pour lui de détruire l'ensemble des structures, professionnelles et sociales, qui limitent l'autonomie de l'individu». (Rosanvallon, 1981, pp. 4445)

1. Nous sommes loin d'une interprétation durkheimienne de la place des corps professionnels dans la société. Celui-ci dans sa Préface à la deuxième édition de La division $d u$ travail social, indique clairement l'importance des corps professionnels comme facteur de médiation de l'appartenance de l'individu à la société. Il se fait de plus l'avocat du renforcement de ces organisations professionnelles devant le fléau de l'anomie.

«Si l'anomie est un mal, c'est avant tout parce que la société en souffre, ne pouvant se passer, pour vivre, de cohésion et de régularité. Une réglementation morale ou juridique exprime donc essentiellement des besoins sociaux que la société seule peut connaître; elle repose sur un état d'opinion, et toute opinion est chose collective, produit d'une élaboration collective. Pour que l'anomie prenne fin, il faut donc qu'il existe ou qu'il se forme un groupe où se puisse constituer le système de règles qui fait actuellement défaut.

$\mathrm{Ni}$ la société politique dans son ensemble, ni l'État ne peuvent évidemment s'acquitter de cette fonction; la vie économique, parce qu'elle est très spéciale et qu'elle se spécialise chaque jour davantage, échappe à leur compétence et à leur action. L'activité d'une profession ne peut être réglementée efficacement que par un groupe assez proche de cette profession même pour en bien connaître le fonctionnement, pour en sentir tous les besoins et pouvoir suivre toutes leurs variations. Le seul qui réponde à ces conditions est celui que formeraient tous les agents d'une même industrie réunis et organisés en un même corps. C'est ce qu'on appelle la corporation ou le groupe professionnel». (Durkheim, c1973, p. VI) 
Glorifier la montée des droits individuels ou au contraire la dénoncer comme une condition de la destruction du social dont il faut restaurer l' "opacité», n'est pas une question à laquelle nous nous attarderons ici. Contentons-nous de constater qu'avec la réforme des professions et, par certains aspects, la réforme de la santé, l'État s'adresse à l'individu-professionnel au-delà de l'entité corporative à laquelle ce dernier est obligé d'appartenir. La reconnaissance de droits individuels aura été un levier important pour imposer aux organismes représentant le corps médical une logique d'action qui leur a permis de répondre aux contestations tant internes qu'externes de leur mode de fonctionnement, et de redevenir pertinents et efficaces dans la défense et promotion des intérêts collectifs ${ }^{2}$.

La thèse étant exposée, nous allons traiter de quelques-uns des éléments empiriques qui ont servi de support à son élaboration et qui sont éclairés en retour par elle. Dans un premier temps, les règles d'accès à la pratique de la médecine en milieu hospitalier de même que le rôle du corps médical dans la gestion hospitalière, retiendront notre attention. À propos de ces éléments, il sera

2. Nous ne prétendons pas que la création de droits individuels a contribué à diminuer le pouvoir de la corporation par exemple, mais plutôt qu'elle a été un moyen pour amener ce pouvoir à s'exercer sur des bases différentes (d'une légitimité de type traditionaliste à une légitimité de type légal-rationnel). Il est probable que les organismes représentant le corps médical auraient changé même en l'absence de ce produit secondaire de la réforme de la santé et des professions; ceci n'empêche cependant pas de considérer les droits individuels comme un adjuvant important. Nous ne prétendons pas non plus qu'aucune trace de légitimité légale-rationnelle n'était présente dans le fonctionnement de ces organismes avant l'intervention de l'État ni qu'aucune trace de légitimité traditionaliste n'y serait actuellement décelable. Il est cependant clair qu'aujourd'hui ces organismes présentent leur place et leur action dans des termes qui recourent peu à des arguments de tradition. Enfin, on peut penser a priori qu'un corps professionnel dont les membres s'adonnent à une prestation de services basés sur l'application d'un savoir scientifique, ne fonctionne de toute manière qu'à la rationalité. Ce serait oblitérer une distinction importante entre acteur collectif et acteur individuel et oublier le caractère éminemment social des conditions dans lesquelles ce savoir se fabrique et s'applique (voir à ce sujet, Eliot Freidson). 
montré 1. que le caractère universaliste (par opposition à particulariste) des nouvelles règles d'accès a permis au corps médical de résoudre une tension croissante en son sein et ce, sur la base de considérations relevant d'une rationalité instrumentale; 2 . que l'imposition de nouveaux critères d'accès qui auraient pu menacer la place occupée par le corps médical dans la définition de ce qu'est un hôpital et des ressources nécessaires à son fonctionnement, n'a pas abouti à un tel résultat mais plutôt à la réaffirmation et à l'élargissement de cette place. Nous prétendons cependant que le rôle du corps médical dans la gestion hospitalière a reposé de moins en moins sur une légitimité de type traditionaliste.

Dans un deuxième temps, les modifications quant aux procédures d'évaluation de la qualité et de la compétence des médecins exerçant en milieu hospitalier, seront examinées de deux manières: tout d'abord, à travers l'étude des législations et réglementations concernant cette évaluation; puis, à travers une étude de cas qui permettra de savoir comment se fait cette évaluation dans un centre hospitalier universitaire au début des années 80 . On s'apercevra alors que les modifications concernant l'évaluation ont contribué à diminuer le caractère arbitraire de celle-ci et à protéger davantage les droits des individus évalués. La conservation du principe de l'évaluation par les pairs est un enjeu crucial pour le degré d'autonomie dont peut jouir un corps professionnel; par ailleurs, cette même évaluation est une source de tension entre pairs surtout si la nature des infractions, les moyens d'en faire la preuve, les recours du pair évalué sont mal définis ou perçus comme comportant une large part de pouvoir discrétionnaire. La création de droits individuels - un produit secondaire de la réforme de la santé et des professions - représente une soupape de sûreté qui permet à un corps professionnel de recréer l'unité nécessaire à la défense des intérêts collectifs. Enfin, la pratique de cette évaluation, lorsque soumise à des procédures universelles 
s'appliquant au sein de toutes les organisations bureaucratiques (ce que sont les hôpitaux), a été l'un des terrains où le corps médical québécois s'est adapté à de nouvelles formes de domination.

\section{Modifications des conditions d'accès à la pratique de la médecine}

Au cours des années 60 , l'identité de l'État québécois comme État moderne se constitue. Dans le domaine de la santé, ce processus laisse les traces suivantes.

La Loi des hôpitaux de 1962 donne aux hôpitaux du Québec le statut d'organismes publics devant rendre compte des fonds de plus en plus importants que l'État y investit. La version de cette loi datant de 1962 donne à la Corporation professionnelle des médecins de même qu'aux deux associations représentant alors les administrations hospitalières, le pouvoir d'élaborer la réglementation afférente à cette loi. Un amendement adopté en 1965 après de multiples manifestations d'opposition de la part du corps médical, leur retire cependant ce pouvoir pour le donner au lieutenant-gouverneur en conseil qui devra néanmoins procéder à la consultation de la Corporation et des deux associations. Ce n'est qu'en 1969 que la réglementation afférente à la loi de 1962 sera promulguée.

Après les premières grèves dans le secteur hospitalier, le gouvernement provincial, passant outre l'autorité des administrations hospitalières, signe les premières conventions collectives (à l'échelle québécoise) avec le personnel hospitalier.

Cette constitution de l'identité de l'État québécois se poursuivra au cours des années 70 dans le domaine de la santé principalement à travers l'adoption d'un régime universel d'assurancesanté; la réforme de la santé et des services sociaux qui soumet l'organisation de la majeure partie de la production des services 
socio-sanitaires à l'appareil gouvernemental; la réforme des législations régissant les corporations professionnelles.

Ces réformes ont été accompagnées d'une diversification des modes de pratique de la médecine depuis le début des années 60 .

La situation au cours de la décennie 60-70 est la suivante. La pratique en milieu hospitalier est devenue un mode de pratique important en partie en raison du fait que les coûts des services fournis en milieu hospitalier ont été couverts par un régime universel d'assurance-hospitalisation. L'accès à la pratique hospitalière se transformait ainsi en un enjeu crucial.

La pratique non hospitalière était de façon prépondérante une pratique en solo. Les services fournis sur une base ambulatoire pouvaient être couverts par des régimes privés d'assurance-maladie. Cette pratique non hospitalière comprenait aussi des visites à domicile en plus des consultations en cabinet.

La situation s'est modifiée au cours de la décennie 70-80. La médecine s'exerçant dans le secteur public (i.e. dans les organisations de distribution de services de santé faisant partie du réseau des Affaires sociales) peut être pratiquée dans un nombre plus important d'organisations: centre hospitalier de courte durée, centre hospitalier de soins prolongés, centre d'accueil, centre local des services communautaires. Même si elles sont plus diversifiées, ces diverses organisations fonctionnent toutes d'après des règles clairement définies dans les textes de loi et de règlement. Dans le secteur privé, il se produit aussi des transformations: la pratique solo tend à diminuer en faveur de la pratique de groupe et en polyclinique. De plus, les visites à domicile ont aussi diminué de façon importante.

Au cours des 20 dernières années, tant la pratique "publique» que la pratique "privée» de la médecine se sont diversifiées. Arrêtons-nous particulièrement à l'accès à la pratique hospitalière parce que ce cas met bien en évidence le contrôle qu'a continué 
d'exercer le corps médical sur cet accès et ce, en dépit des modifications qui ont pu survenir depuis 1962. En effet, le gouvernement québécois a tenté à plusieurs reprises et de diverses manières de restreindre l'exercice de la médecine en milieu hospitalier et ce, depuis le début des années 70 surtout. L'idée était de diminuer l'importance trop grande accordée à la guérison des épisodes aigus de maladie de manière à orienter davantage le système de santé vers une médecine préventive et communautaire. Voici quelques dates marquant ces tentatives de contrôler l'accès à la pratique hospitalière:

- En 1962, les centres hospitaliers (CH) deviennent des organismes publics mais la réglementation afférente à cette loi de 1962 n'est promulguée qu'en 1969. Dans celle-ci, on retrouve qu'un médecin voulant pratiquer dans un $\mathrm{CH}$ donné, doit fournir des preuves de qualifications, titres, compétence de même que de personnalité et caractère compatibles avec l'emploi.

- D'après la loi de 1971 qui marque l'instauration de la réforme de la santé et des services sociaux, les raisons de refuser la candidature d'un médecin doivent renvoyer à la compétence scientifique, aux qualifications et au comportement.

- En 1974, le Ministre Forget tente par l'ajout d'un amendement («eu égard aux exigences propres au $\mathrm{CH}$ ») d'imposer le nombre de médecins déjà admis ou la quantité de ressources disponibles comme raisons de refus. En vain.

- À partir de 1978, le nombre de médecins déjà admis dans un CHU (centre hospitalier universitaire) peut être invoqué comme raison de refus d'une nouvelle candidature.

- En 1981, cette raison est étendue à tous les $\mathrm{CH}$ pour les nouvelles candidatures et non pas pour le renouvellement des candidatures déjà acceptées. Au même moment, le Conseil des médecins et 
dentistes $^{3}$ de l'hôpital acquiert le droit d'être consulté sur le plan d'organisaion (y inclus, la quantité des effectifs) de l'hôpital.

Après avoir aboli l'existence de chasses-gardées dans les hôpitaux en ouvrant la pratique hospitalière à presque tout médecin qui en faisait la demande (tout refus écrit ne devant en effet reposer que sur un nombre défini de raisons), le gouvernement a tenté à plusieurs reprises d'imposer sa logique de rationalisation des ressources au cours des années 70, logique qui lui dictait de restreindre ce droit de pratique en milieu hospitalier pour arriver à mieux distribuer les effectifs médicaux parmi les hôpitaux du territoire québécois. Ce n'est qu'en 1978 que ces restrictions purent être appliquées et encore, dans le seul cas des CHU. Or, dès cette année-là, le Conseil des médecins et dentistes de l'hôpital acquit le droit d'être consulté sur le plan d'organisation du CHU avant que celui-ci ne soit adopté. En 1981, la possibilité pour tout $\mathrm{CH}$ d'invoquer le nombre des médecins déjà présents comme motif de refus d'une nouvelle nomination, est acquise. Depuis ce moment-là, le Conseil des médecins et dentistes de tout hôpital doit être consulté sur le plan d'organisation clinique; de plus, à partir du début des années 80 , le chef de département devient le gestionnaire «local» des ressources. Ce qui, à première vue, apparaît comme la victoire d'une logique rationalisatrice qui aurait mis plus de dix ans à s'imposer, doit être relativisé puisqu'en échange de ces restrictions, le corps médical d'un $\mathrm{CH}$ composant le $\mathrm{CMD}$ pourrait, par exemple, arguer que les effectifs prévus au plan d'organisation pour tel ou tel département clinique sont insuffisants. Il est probable cependant qu'aussi longtemps que ce plan ne

3. Le Conseil des médecins et dentistes d'un hôpital (on y a ajouté depuis les pharmaciens) réunit les médecins et dentistes exerçant leur profession dans ce centre hospitalier. Il s'agit d'un organe qui est responsable devant le conseil d'administration de l'hôpital des questions de qualité des actes médicaux et de compétence des médecins. Cet organe doit aussi être consulté à propos de diverses décisions concernant l'organisation clinique des services médicaux. 
contiendra pas de réduction des effectifs présents, les médecins déjà admis à la pratique ne créeront pas d'opposition systématique au gel des effectifs dans les $\mathrm{CH}$ bien pourvus, gel qui, de toute façon, constitue un moyen de limiter la concurrence.

La création à partir de 1969 d'un «droit individuel à la pratique hospitalière» à travers l'énonciation d'un nombre défini de critères explicites a servi à briser l'emprise de l' «establishment » médical dans les différents hôpitaux. L'exercice de ce droit individuel a cependant vite trouvé sa limite dans une volonté de contrôler la croissance des coûts et d'assurer une distribution équitable des effectifs. Les restrictions récentes à l'exercice de ce droit individuel ont par ailleurs été l'occasion pour le corps médical des hôpitaux comme entité groupale de retrouver des pouvoirs de gestion plus étendus à l'intérieur de ces lieux socialisés de production des services de santé. Ces pouvoirs de gestion ne peuvent cependant plus être exercés au nom de la tradition ou sur la base d'affinités de vues morales, politiques ou autres; l'adéquation des moyens aux fins poursuivies ou la rationalité instrumentale s'est, entretemps, fermement installée.

La place du corps médical dans la gestion hospitalière a aussi évolué au cours de la période 60-80. Si nous comparons 1 . les fonctions du directeur médical, du Bureau médical, des comités qui y sont rattachés, et des chefs de département des années 60 avec 2. les fonctions du directeur des services professionnels, du CMD, de ses comités, et des chefs de département telles qu'elles étaient au début des années 1970 et 3. telles qu'elles sont devenues au début des années 1980 , nous pouvons constater un retour du balancier. En effet, autant le corps médical était, dans les années 60, présent dans la gestion de l'hôpital à travers ses privilèges et à travers tous ces nouveaux mécanismes par lesquels on tentait, avant le texte de loi, de contrôler de quelque manière la pratique médicale et qui servaient en fait à influencer le cours des choses 
dans l'hôpital (ex.: comité mixte médico-administratif), autant on a cherché à distinguer au début des années 1970 la ligne d'autorité médicale de la ligne d'autorité administrative (ex.: disparition de ce comité) et à circonscrire l'influence du corps médical à la seule supervision des services médicaux. Il est donc étonnant de voir, au début des années 80, que des fonctions administratives de plus en plus importantes sont confiées au chef de département clinique et au DSP et que des fonctions-conseil sont attribuées au CMD. Nous verrons plus loin comment il est possible d'interpréter ce retour du balancier.

Pour l'instant, passons à un autre exemple, celui du contrôle de la qualité des actes médicaux et de la compétence en milieu hospitalier. Voici le portrait de ce contrôle de la pratique médicale tel qu'il a pu être établi pour 1983 (voir Figure 1).

\section{Modifications de l'évaluation de la qualité et de la compétence en médecine}

\section{Modifications législatives et réglementaires}

Les constatations et conclusions rapportées ici reposent sur un examen de textes législatifs et réglementaires entre 1962 et 1983 de même que sur l'examen de procès-verbaux des comités d'évaluation médicale et dentaire d'un centre hospitalier universitaire sur une période d'un an.

Les principales structures mises en place au sein des hôpitaux pour surveiller la qualité/compétence sont les suivantes:

Décennie 60

Directeur médical

Bureau médical

- comité des créances

- comité d'appréciation des actes médicaux

Chef de département
Décennie 70

Directeur des services professionnels (qui est toujours un médecin)

Conseil des médecins et dentistes (CMD)

- comité d'examen des titres

- comité d'évaluation médicale et dentaire

Chef de département clinique 
(1)

Division de

l'agrément, MAS

(3)

- Conseil des médecins et dentistes

- Comité d'évaluation médicale et dentaire (tissus, dossiers, mortalité maternelle et infantile), comités de pharmacologie, séjour, examen des titres

- Directeur des services professionnels (DSP)

- Chef de département clinique

(1) L'article 21 de L.Q. 1981, c. 22 prévoit des possibilités de transmission, par la RAMQ, 1. des profils collectifs de pratique au CMD d'un établissement et 2 . des profils individuels de pratique (sans identité) aux chefs des départements cliniques des $\mathrm{CH}$.

(2) De l'étude de Deschamps et al., 1978, nous pouvons retenir que: 1. des 665 poursuites judiciaires intentées entre 1968 et 1977 , 334 étaient encore pendantes au moment de l'étude; 2. des 327 poursuites terminées, 214 avaient donné lieu à des règlements hors Cour; dans 35 cas, le plaignant s'était désisté; 78 poursuites ont donné lieu à un jugement de la Cour; 3 . dans ce dernier cas (78 jugements de la Cour), le plaignant a eu gain de cause dans $38,8 \%$ des poursuites (Données manquantes dans 4 cas).
Recours des patients:

- poursuites judiciaires ${ }^{2}$

- plaintes à la

- corporation professionnelle

- au DSP d'un $\mathrm{CH}$

- au CRSSS
- profils individuels et collectifs

SOINS DE $1^{\text {ère }}$ LIGNE

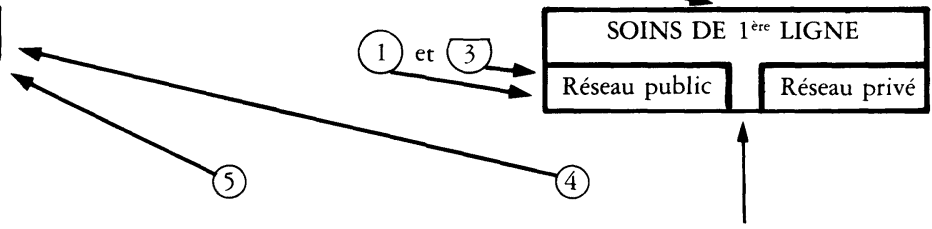

Conseil canadien d'agrément des hôpitaux

(Comités d'inspection professionnelle et de discipline) 
Une première constatation a trait à la conservation du principe corporatif du contrôle de la qualité des services médicaux par les pairs même lorsque ces services sont fournis dans un cadre bureaucratique hétéronome. En dépit des modifications survenues au cours de la période 1971-1981 et qui, à la surface, paraissent menacer ce contrôle, la décennie 70 aura été en fait le théâtre d'un changement des bases de légitimation de l'action des diverses instances représentant le corps médical au sein de l'hôpital. En effet, le combat apparent entre rationalisateurs bureaucrates et monopoleurs professionnels n'est pas une lutte entre ennemis mais plutôt entre fractions d'un même camp. Les rationalisateurs bureaucrates ayant mis de l'ordre dans ce qui n'apparaissait que pouvoir discrétionnaire, arbitraire, privilèges, ils sont maintenant en mesure de redonner aux monopoleurs professionnels - non pas un pouvoir qu'ils n'ont jamais perdu - mais un rôle conforme à des modèles de décision et d'action, à des normes qui sont plus adaptées à l'évolution récente de la société québécoise dans son ensemble. La supervision, l'appréciation, l'évaluation des actes médicaux en ressortent "modernisées", les procédures, les liens entre instances, les responsabilités respectives étant clairement définis. Après une décennie d'apprentissage pendant laquelle les médecins ont pu s'habituer à ces nouveaux modèles de fonctionnement en prenant leurs propres mécanismes d'évaluation comme terrain d'essai, ils sont maintenant en mesure d'élargir le champ de leurs responsabilités à la gestion des ressources d'un département, par exemple. L'avantage principal de cette nouvelle définition de la situation pour les rationalisateurs bureaucrates réside principalement dans le fait que, les médecins déterminant de toute façon par leurs habitudes et leurs comportements quotidiens la destinée de cette organisation bureaucratique qu'est le centre hospitalier, ils seront dorénavant tenus eux aussi responsables de la 
gestion du $\mathrm{CH}$ devant l'opinion publique et devront vraisemblablement définir et évaluer la qualité à la mesure de ressources de plus en plus limitées. Si les monopoleurs professionnels continuent d'être les seuls définisseurs de la qualité et les seuls dépositaires de l'autorité permettant de corriger des abus - et on a pu voir à maintes reprises que la Corporation professionnelle et les syndicats se sont assurés de la préservation de ce monopole - , alors ils pourront par le fait même être de meilleurs complices des rationalisateurs bureaucrates dont le plus grand défi au cours des années 80 consiste à gérer la pénurie. Ce n'est peut-être même que dans la mesure où les monopoleurs professionnels créent l'impression qu'ils luttent contre les rationalisateurs bureaucrates pour défendre ce contrôle de la qualité et de la compétence - ce qui augmente la crédibilité de leur avis sur cette qualité/compétence -, qu'ils peuvent le mieux venir en aide aux rationalisateurs bureaucrates dans cette gestion de la décroissance.

Faut-il pour autant en conclure que rien n'a changé et que nous sommes de retour à la case départ? Il nous semble que non: il n'est pas indifférent, pour le fonctionnement d'une société, qu'une partie de plus en plus grande de ses membres doivent invoquer des arguments faisant appel à la rationalité en finalité plutôt qu'à la tradition, à la transmission des droits acquis de génération en génération ou aux affinités de vues morales, politiques ou autres, pour justifier leur action, leur prise de position. La réforme en santé du Québec a permis aux groupes professionnels - du moins, à la profession médicale - (ou les a obligés) de faire l'apprentissage de cette rationalité instrumentale qui, une fois complété, leur permet de maintenir une position sociale enviable par rapport aux autres groupes occupationnels.

Revenons pour un instant à la diversification des modes de pratique qui s'est produite au cours de cette décennie. Les modes de pratique sont certes importants puisqu'ils exercent une influence 
sur la configuration du produit offert par le professionnel. Mais ils sont aussi importants à un autre titre: leur potentiel à servir de «terrain d'apprentissage» (ou lieux de confrontation) de la rationalité instrumentale pour un nombre grandissant de professionnels. Le déclin de la pratique en solo, la fréquence importante de l'utilisation des lieux socialisés de production des services médicaux par les professionnels-médecins confrontent ces derniers à une logique en action fort différente de celle à laquelle l'École de médecine et l'Association locale de médecine ont pu les initier. La marque laissée sur eux lors de leur passage dans ces modes de pratique pourrait être éphémère si, par ailleurs, la réforme des professions n'avait elle aussi permis aux corporations professionnelles de devenir de tels terrains d'apprentissage, comme nous le verrons au dernier point de cette section.

Une deuxième constatation à tirer de la comparaison entre la décennie 60 et la décennie 70 quant aux diverses instances de contrôle de la pratique médicale en milieu hospitalier, concerne la reconnaissance très étendue des droits individuels du médecin (au-delà du pouvoir de facto du corps médical dans son ensemble au sein de l'hôpital). Cette reconnaissance se manifeste, entre autres, par le fait 1 . que le médecin essuyant un refus de nomination dans un $\mathrm{CH}$ peut en appeler de cette décision devant un organisme extérieur au $\mathrm{CH}$ (la Commission des affaires sociales par opposition au comité exécutif du Bureau médical ou au conseil d'administration dans la réglementation de 1969); 2. que les motifs possibles de refus de nomination et de renouvellement sont clairement identifiés et qu'un d'entre eux doit servir de justification au rejet écrit d'une candidature (on passe, par exemple, des motifs tels que "caractère ou personnalité» à des motifs ayant trait au «comportement» qui se manifeste dans l'inconduite ou l'inobservance des règlements); 3. que le médecin n'a plus besoin d'autorisation écrite pour exercer dans plusieurs $\mathrm{CH}$; 4. que le médecin ne peut plus être la cible 
d'une sanction si un acte ou une omission de sa part ne sont pas des infractions à un règlement explicite (par opposition à des coutumes ou moeurs) (C.A.S. 1977, 718, cité dans Comtois, 1982, pp. 25-26). Ce qui amène $S$. Comtois à conclure, au terme de sa revue du contrôle de l'exercice de la médecine en milieu hospitalier:

«... on doit reconnaître que d'immenses progrès ont été réalisés. On aura en effet réussi, à l'aide de l'élaboration de critères de sélection et l'octroi de garanties procédurales à sortir de l'arbitraire le processus de sélection du personnel médical exerçant en milieu hospitalier et en conséquence, à protéger à la fois les intérêts des médecins dûment qualifiés et compétents et les intérêts des bénéficiaires des services de santé. Dans l'ensemble la principale lacune du système aura été le déséquilibre entre la reconnaissance d'un quasi-droit pour le médecin à exercer sa profession en milieu hospitalier et l'impossibilité pour le centre hospitalier de tenir compte de ses besoins légitimes». (S. Comtois, 1982, pp. 29-30)

"On reconnaîtra cependant que les divers mécanismes de contrôle institués par la Loi sur les services de santé et les services sociaux favorisent la protection des intérêts des médecins et ce, au détriment du centre hospitalier que l'on a dépouillé d'une large part de son autonomie et de son autorité en matière d'organisation professionnelle». (S. Comtois, 1982, p. 9)

Soustraction donc de l'individu-médecin à l'emprise de l'organisation professionnelle et à celle de l'organisation bureaucratique qui doit respecter les règles explicites qu'elle se donne pour guider son fonctionnement. Cette soustraction s'accomplit d'ailleurs selon des formes qui ne sont pas étrangères à l'imposition de la rationalité instrumentale. Le fait d'accorder des droits à l'individu-médecin, droits qu'il peut défendre selon des procédures clairement préétablies, ne constitue-t-il pas un moyen privilégié d'inciter le professionnel à participer de cette rationalité et à en devenir un défenseur? Cette deuxième constatation serait, par conséquent, à relier à la première.

Une troisième constatation porte sur la définition des "privilèges ${ }^{4}$ accordés lors de la nomination d'un médecin ou de son

4. Ces privilèges indiquent surtout quels sont les actes médicaux qui ne peuvent être posés par un médecin exerçant dans un centre hospitalier. 
renouvellement en milieu hospitalier, et de leur influence sur la définition de l'exercice de la médecine, source d'identité importante pour l'ensemble du corps professionnel. L'insertion de la pratique médicale dans le milieu hospitalier ne peut certes pas être comparée au cas d'un bon nombre d'autres occupations qui dépendent d'une organisation pour s'exercer. En effet, dans le cas des médecins, l'hôpital-employeur ne peut pas déterminer le niveau de qualification de la main-d'œuvre médicale ni accorder un emploi aux médecins sur la base d'une hiérarchisation des compétences ou qualifications qui seraient identifiées à l'occupation de divers postes dans la structure de l'entreprise. Cette situation habituelle pour la plupart des occupations s'oppose à la situation où les fournisseurs de services dessinent eux-mêmes, au sein de l'organisation-employeur, les cases où ils auront droit de se loger. Mais même dans ce dernier cas, l'organisation laisse son empreinte sur l'identité professionnelle à travers, par exemple, la définition des privilèges, l'appartenance à un département de médecine par opposition à un département de chirurgie ou à un département de santé communautaire, l'appartenance à un service de recherche par opposition à un service clinique, etc. Or l'appartenance à diverses parties de l'organisation fonde des points de vue différents sur la place, la destinée du corps médical dans l'ensemble de la société, dans ses rapports avec l'État, etc. Elle constitue par conséquent une menace éventuelle à l'unité de l'organisation professionnelle. Comment celle-ci peut-elle continuer à représenter les intérêts des chercheurs par opposition aux cliniciens, des chirurgiens par rapport aux internistes, etc. ? D'où le paradoxe suivant: l'organisation professionnelle pourra d'autant mieux garder un degré minimal de cohésion nécessaire pour l'action efficace si son emprise sur ses membres n'est pas omniprésente. Or la reconnaissance des droits des individus-médecins tant au sein de l'organisation-employeur qu'au sein de l'organisation professionnelle, reconnaissance qui 
est le résultat de la réforme en santé et de la réforme des professions au Québec, permet justement aux intérêts divergents fondés sur l'occupation de places différentes dans divers milieux organisés de pratique, de s'exprimer sans que cela ne menace l'unité de l'organisation professionnelle. Aussi paradoxal que cela puisse paraître, l'instauration des droits individuels comme frontière à l'omnipotence de l'organisation professionnelle tant au sein qu'en dehors des organismes-employeurs, constitue une condition de succès du projet professionnel.

\section{Une étude de cas}

L'étude de cas menée à partir des procès-verbaux des comités d'évaluation d'un centre hospitalier universitaire nous amène à faire les constatations suivantes. Les dossiers étant assez souvent mal tenus - et ceci est un commentaire fréquent de presque toutes les instances - il est difficile de procéder à une évaluation sérieuse puisque fondée sur de tels dossiers. De plus, le processus de délégation des responsabilités est tel qu'on aboutit souvent à la situation suivante: ceux qui doivent procéder à l'évaluation sont aussi ceux qui posent les actes qui peuvent devenir l'objet d'une évaluation. Le même processus de délégation implique cependant que chaque instance inférieure doit rendre des comptes à l'instance supérieure. Le sérieux avec lequel le département ou le service s'acquitte de ses tâches, dépend certes d'un bon nombre de facteurs (ex. : rôle du chef de département) mais on peut estimer qu'il varie aussi selon la pression que l'instance supérieure exerce sur lui. Or, les sous-comités qui doivent faire rapport au comité central d'évaluation, conçoivent leur rôle de façon très différente. De plus, les échanges à un même niveau hiérarchique (interservices ou inter-sous-comités) sont très peu fréquents et, lorsqu'ils surviennent, ils donnent lieu à des réactions défensives la plupart du temps. Enfin, le comité central qui fait rapport au CMD et 
au DSP (le DSP faisant partie de l'exécutif du CMD), exerce surtout un rôle de surveillance et d'instance à laquelle les souscomités doivent se rapporter.

Bref, si contrôle de qualité et/ou de la compétence il y a, il n'est certes pas manifeste dans les procès-verbaux consultés, sauf exception. On pourrait arguer qu'il suffit que les médecins pratiquant en $\mathrm{CH}$ connaissent l'existence de ces diverses instances pour s'auto-contrôler, et qu'il suffit que cette existence se manifeste à de rares occasions par l' «attention suivie» dont la pratique d'un médecin peut devenir l'objet pour que s'institue un auto-contrôle efficace. Cela est possible et nous renvoie à la difficile question: les moyens adoptés (i.e. les formules d'encadrement propres au $\mathrm{CH}$ ) permettent-ils d'atteindre les fins poursuivies? Cette question demeure sans réponses concluantes tant que 1. il n'existe pas de mesure indépendante de la qualité/compétence qui permettrait de comparer, pour un même niveau de qualité, le fonctionnement de ces mécanismes d'encadrement dans divers $\mathrm{CH}$ semblables quant au type de services offerts; 2 . l'on se fie aux procès-verbaux pour avoir une image fidèle de ce qui se déroule dans ces comités de toutes sortes.

Mais cette façon d'analyser les résultats de cette étude de procès-verbaux n'en est qu'une; de plus, elle n'est pas distinctement sociologique puisqu'en effet gestionnaires et consommateurs se posent cette question de l'influence de ces mécanismes sur la qualité des services fournis. Si nous passons à un autre niveau d'analyse, nous pouvons nous arrêter à d'autres phénomènes se rattachant à l'influence qu'exerce l'insertion de la pratique médicale dans le milieu hospitalier, sur divers aspects de celle-ci. Plus spécifiquement: comment les membres du corps médical placés devant une obligation, celle de l'auto-évaluation de leurs pratiques, s'adaptent-ils au schéma moyens-fins et au fait qu'ils doivent rendre des comptes à des instances qui n'émanent pas de leur 
organisation professionnelle et ce, en utilisant un schéma qui est propre à ces dernières? Tout d'abord, il faut constater que leurs activités d'auto-évaluation se limitent strictement aux obligations que leur crée la réglementation et, de plus, aux aspects les plus visibles de celles-ci (ex.: décès et complications). Ils cèdent par ailleurs aussi à des obligations dont le respect ou le non-respect est moins visible (ex.: obligation de juger de la qualité des services) en procédant à quelques évaluations par critères objectifs. De plus, ils rendent compte de ces activités en donnant rarement à une personne extérieure l'information nécessaire pour juger de l'efficacité de ces activités (ex. : les procès-verbaux donnent rarement la portion des cas de décès ou des cas avec complications sur le total des cas traités). Enfin, les médecins étudiés ont aussi appris à se renvoyer la balle de sous-sous-comité en sous-comité en comité central et à enrober leur refus d'agir dans la définition de juridictions.

Par ailleurs, un bon nombre d'autres indications montrent qu'en dépit d'un certain apprentissage de la rationalité instrumentale et de ses perversions, il existe encore des ratés (à preuve la nécessité d'avertir tel chef de département qu'il doit séparer affaires administratives et évaluation). Ces ratés s'expliquent en partie par la persistance d'habitus corporatifs au cœur de l'institution hospitalière, habitus qui se manifestent surtout lorsque des confrères d'un même service ou département sont contraints de s'évaluer les uns les autres. Il demeure non éthique de se critiquer entre collègues et ceux-ci ne se décideront à agir que sur l'impulsion qui leur sera donnée par une instance qui leur est extérieure.

Au terme de cette étude du contrôle de la qualité/compétence, il est important de souligner qu'il ne faut pas minimiser l'importance des changements législatifs et réglementaires survenus depuis 1962 dans l'encadrement de la pratique médicale en milieu hospitalier. Cet encadrement n'est certes plus le même: il prend plusieurs formes, passe par plusieurs instances. Cette quasi-omniprésence 
ne doit cependant pas nous faire conclure que la pratique hospitalière est désormais soumise aux diktats des administrations profanes (et par opposition à religieuses, et par opposition à professionnelles) des $\mathrm{CH}$. Cette évolution de la législation et réglementation n'a pas mené à des changements où tout serait noir d'un côté, et blanc de l'autre. Nous soutenons plutôt que cette évolution a eu pour résultat certes d'encadrer davantage la pratique hospitalière mais qu'elle a aussi et en même temps constitué une occasion pour le corps médical de se mettre à l'heure du fonctionnement des autres institutions envahies par la rationalité instrumentale.

\section{L'évaluation au sein de la Corporation}

L'hôpital n'est pas le seul cadre possible de la pratique médicale. La Corporation des médecins a pour tâche de contrôler la qualité de cette pratique, y compris en dehors des milieux hospitaliers. Nous n'allons pas rendre compte ici de la façon dont cette Corporation s'acquitte de cette tâche (voir Couture, 1986). Nous allons plutôt nous limiter à quelques réflexions sur le rôle d'un de ces organes de contrôle, le comité de discipline de la Corporation.

La réforme des professions en 1973 a eu comme effet principal d'imposer à toutes les corporations reconnues un cadre uniforme définissant les devoirs, privilèges, fonctions, pouvoirs, procédures de fonctionnement de ces corporations.

Ainsi, les comités de discipline des corporations sont encadrés par un ensemble de règles applicables à toutes. Ceci retire aux corporations l'exercice d'un pouvoir discrétionnaire en matière de gestion de la délinquance professionnelle. Par exemple, la Corporation des médecins n'a plus, depuis la réforme des professions, le pouvoir de réglementer sur les procédures à adopter dans le traitement d'une plainte. 
Divers éléments de cette réforme concernant la définition de ce qui constitue une infraction et une sanction, la composition du comité de discipline, les procédures d'appel sur un verdict rendu, etc. sont autant de garanties du respect des droits des individus-médecins et autant de limites à l'arbitraire d'une entreprise de police de mœurs, dans laquelle pourrait se lancer une entité corporative à laquelle il faut appartenir si l'on veut exercer son métier.

Dans le cas du fonctionnement interne de la Corporation des médecins, la création et la garantie par l'État des droits individuels ont été là aussi la base d'un nouveau départ pour la crédibilité de la Corporation aux yeux de ses propres membres. Celle-ci était en effet menacée d'implosion au cours des années 60 alors que les luttes intestines entre d'une part les médecins bien établis soit dans les hôpitaux soit au sein de la Corporation et, d'autre part, les médecins nouveaux à la recherche d'une place au soleil, ne pouvaient plus être contrôlées à l'interne. C'est d'ailleurs au cours de ces années qu'on assiste à la constitution de deux syndicats professionnels (celui des omnipraticiens et celui des spécialistes), ce qui peut indiquer jusqu'à quel point une partie du corps médical québécois remettait en question la légitimité de cette Corporation.

Nous n'irons pas jusqu'à affirmer que c'est grâce à l'intervention de l'État sous forme de modifications au droit professionnel que la Corporation des médecins a été en mesure de survivre. Mais il est intéressant d'examiner en rétrospective quelle contribution cette intervention a pu apporter à l'efficacité renouvelée de l'action de cette Corporation.

\section{CONCLUSION}

Nous ne prétendons pas que rien n'a changé au Québec en ce qui concerne la profession médicale et son système de santé. 
Au contraire: nous avons indiqué quelle était la nature des modifications apportées tant à un niveau superstructurel (les règles régissant divers domaines de l'exercice de la médecine) qu'à un niveau infrastructurel (effectifs, modes de pratique, etc.). Nous prétendons seulement que l'impact de ces modifications n'a pas consisté (ou pas encore - cela demeure ouvert) à faire perdre au corps médical québécois sa place de choix au sein du monde de la santé ou au sein de la société québécoise. Qui dit changement dit aussi réaction au changement de la part de ceux qui sont visés par celui-ci. Or, les corps d'occupation organisés sur le modèle professionnel et, surtout, les professions plus anciennes (i.e. dont la naissance n'est pas directement reliée aux réalisations du capitalisme et aux interventions de l'État prenant en charge un domaine de l'activité sociale) possèdent des ressources importantes pour réorienter le changement dans une direction qui ne les menace pas dans leur existence. Nous soutenons que, dans le cas de la profession médicale québécoise, les changements provoqués en partie par l'intervention de l'État, ont été l'occasion pour cette profession de renouveler les bases de la légitimation de sa place et ses activités et ce, dans le sens d'une «modernisation», d'une plus grande adaptation de son fonctionnement à l'ensemble des autres institutions québécoises. Ces changements doivent donc être considérés non seulement du point de vue de leur(s) initiateur(s) mais aussi de leur(s) récepteur(s).

L'idée que la position occupée par un groupe dans le monde du travail (ou le procès de production) détermine à elle seule la place occupée par ce groupe dans la société, peut difficilement s'appliquer dans le cas des professions et du corps médical québécois. L'intendance ("gubernatorial power») assurée par le personnel infirmier, la multiplication des occupations paramédicales, l'automatisation d'un bon nombre de procédés diagnostiques, la standardisation des pratiques médicales à travers l'adoption de protocoles 
thérapeutiques, tous ces éléments contribuent à diminuer l'importance du professionnel-médecin dans la division du travail sanitaire. Pourtant, cela ne contribue pas à diminuer la place de ce corps professionnel dans la définition de la santé et de la maladie, ni à obliger celui-ci à rendre compte de ses activités aux profanes. Il faut le constater: c'est en s'appuyant aussi sur des ressources situées hors de l'univers de travail que le corps médical québécois réussit à s'assurer une position de domination dans la division du travail. Les rapports sociaux globaux déterminent les rapports sociaux qui se nouent et se dénouent dans les activités de production.

Cette constatation nous indique qu'il faudrait examiner soigneusement quelles sont les conditions du succès que rencontrent divers groupes professionnels dans leur résistance au changement ou dans sa réorientation. Nous avons centré notre attention sur les activités de l'organisation professionnelle (syndicats et Corporation) comme un des facteurs de ce succès. Comment s'y prendelle pour défendre les intérêts de ses membres, pour leur assurer une position de domination ou pour, éventuellement, élargir leur domaine d'influence? Une brève réflexion sur ces questions nous permet de penser que la différenciation accrue au sein du corps médical québécois qui résulte tant de l'insertion des pratiques médicales dans divers modes de pratique que de la vulnérabilité plus grande des cohortes de jeunes médecins à certaines contraintes du marché de l'emploi, ou du degré variable de standardisation (pouvant, elle, mener à un processus de déqualification/surqualification) des pratiques médicales, peut causer des difficultés croissantes à cette organisation professionnelle et influencer sa capacité d'action comme organisation politique.

Souligner que le médecin de CLSC est différent du médecin d'un centre hospitalier sur-spécialisé, qu'un médecin de santé communautaire ou de santé au travail n'est pas un néphrologue 
ou un médecin de médecine nucléaire, que les médecins plus jeunes ne sont plus soumis aux mêmes conditions du marché que les plus âgés, que la médecine de Montréal n'est pas celle de Rouyn-Noranda, contribue d'abord à se débarasser d'une image d'unité que tout corps professionnel veut et réussit à projeter. Tous les médecins ne sont pas atteints, par exemple, de la même façon d'une baisse éventuelle de pouvoir d'achat et ne possèdent pas non plus les mêmes moyens pour y faire face. De plus, souligner ces différences nous amène à réfléchir sur l'importance de l'organisation professionnelle dans la défense des privilèges du corps médical et sur les difficultés que cette différenciation peut causer à cette organisation dans la présentation d'un front unifié face à l'extérieur. En effet, si la diversification des modes de pratique, l'insertion des pratiques médicales dans une variété de cadres bureaucratiques n'entraînent pas une prolétarisation des professionnels, elles risquent de fonder des perspectives fort différentes sur la définition de la santé ou de la place des médecins dans une société. Comment les organisations défendant les intérêts des professionnels-médecins s'y prennent-elles pour faire face à cette diversité?

Nous avons indiqué que la diversification des modes de pratique, la bureaucratisation d'au moins un mode important de pratique médicale (celui de la pratique hospitalière) et l'institution de nouvelles règles de fonctionnement des corporations professionnelles en matière de discipline et d'inspection, avaient aussi eu comme effet de protéger de façon claire les droits individuels des médecins. Cette consécration des droits individuels - un des résultats paradoxaux de la réforme en santé et de la réforme des professions - permet justement une expression minimale de dissidence, reflet des conditions de travail spécifiques de différents segments du corps médical. Nous soutenons de plus que cette «expression de la dissidence» ou la garantie du droit à cette 
expression est une condition importante du maintien d'une unité suffisante au sein du corps médical québécois. Le maintien de cette unité est d'autant plus crucial qu'un des effets de l'instauration d'un régime public d'assurance-santé et de l'intervention de l'État qui se traduit par une bureaucratisation croissante des modes de pratique, a été de déplacer sur la place publique un bon nombre de questions qui relevaient auparavant presqu'exclusivement du domaine de l'organisation professionnelle.

\section{RÉFÉRENCES}

COMTOIS, S., "Le contrôle de l'exercice de la médecine en milieu hospitalier ", Administration bospitalière et sociale, $1^{\text {ère }}$ partie: janvier-février 1982 , p. $4-10 ; 2^{\mathrm{e}}$ partie: marsavril 1982 , p. $23-30$.

COUTURE, Denise, «La réforme du droit professionnel au Québec: le cas de la corporation professionnelle des médecins", Sociologie et sociétés, XVIII, 1, 1986, p. 97-112.

DESCHAMPS, Pierre, Analyse statistique des poursuites judiciaires en responsabilité civile intentées contre les professionnels de la santé et les établissements de santé du Québec entre le $1^{\text {er }}$ janvier 1968 et le 31 décembre 1977, Montréal, McGill University, 1978.

DURKHEIM, Émile, De la division du travail social, Paris, P.U.F., c 1973.

ROSANVALLON, P., La crise de l'État-Providence, Paris, Seuil, 1981.

WEBER, Max, Économie et société, Paris, Plon, c 1971. 